\title{
Nonclassicality in the statistics of noncommuting observables: Nonclassical states are more compatible than classical states
}

\author{
Alfredo Luis* \\ Departamento de Óptica, Facultad de Ciencias Físicas, Universidad Complutense, E-28040 Madrid, Spain
}

(Received 21 November 2010; revised manuscript received 6 March 2011; published 8 July 2011)

\begin{abstract}
We study nonclassicality in the product of the probabilities of noncommuting observables. We show that within the quantum theory, nonclassical states can provide larger probability product than classical states, so that nonclassical states approach the nonfluctuating states of the classical theory more closely than classical states. This is particularized to relevant complementary observables such as conjugate quadratures, phase and number, quadrature and number, and orthogonal angular momentum components.
\end{abstract}

DOI: 10.1103/PhysRevA.84.012106

PACS number(s): 03.65.Ta, 03.65.Ca, 42.50.Dv, 42.50.Ar

\section{INTRODUCTION}

In this work, we combine two basic issues of the quantum theory: complementarity and nonclassicality [1]. Complementarity implies that if the probability of a property is concentrated around a single value, the probability distribution of complementary observables spread out and nowhere take significant values. This is to say that the product of probabilities of complementary events cannot be large since the increase of one of them implies the decrease of the other.

Complementarity is usually invoked as a nonclassical feature, since in the classical theory any two events can have probabilities as close at one as desired simultaneously by suitably preparing the state of the system (this is by approaching the nonfluctuating states allowed within the classical theory). Thus, from first intuition we might expect that the so-called classical states of the quantum theory will achieve larger product of probabilities for complementary variables than the nonclassical ones, as illustrated by the upper panel of Fig. 1. However, in this work we show that this is not the case and nonclassical states can be closer than the classical states of the quantum theory to the nonfluctuating states of the classical theory, as illustrated by the lower panel in Fig. 1.

To demonstrate this, we look for the maximum product of probabilities for two noncommuting events allowed for the classical states of the quantum theory (Sec. II). This provides a nonclassicality test: if the product of probabilities surpasses the classical bound then the state is nonclassical. (In some previous works where we have derived nonclassical tests via the violation of classical bounds on the probability of single and multiple outcomes of the same observable [2-4].) We show that there are nonclassical states surpassing the bound in different contexts, such as for conjugate quadratures (Sec. III), phase and number (Sec. IV), quadrature and number (Sec. V), and orthogonal angular-momentum components (Sec. VI).

Several other interesting nonclassical tests have already been introduced in the past [5]. In comparison with them, the two main distinctive features of the approach presented here are: (i) this deals directly with probabilities instead of moments or any other more involved data processing. Thus, these tests are extremely simple and robust against practical imperfections, and (ii) this involves the statistics of two

\footnotetext{
*alluis@ fis.ucm.es; http://www.ucm.es/info/gioq
}

observables within a single-mode configuration, instead of just one observable in more standard approaches. This discloses nonclassical properties in the product of probabilities that may not be displayed by the single statistics of the individual observables. Some other paradoxical traits in the statistics of two complementary observables can be found in Ref. [6].

\section{CLASSICAL UPPERBOUND ON THE PRODUCT OF PROBABILITIES OF NONCOMMUTING EVENTS}

Let us consider two events, referred to as $\mu$ and $\nu$. Their individual probabilities when the state of the system is $\rho$ are

$$
p(k \mid \rho)=\operatorname{tr}[\Delta(k) \rho], \quad k=\mu, \nu,
$$

where $\Delta(k)$ are elements of different positive operator valued measures, with $\Delta^{\dagger}(k)=\Delta(k)$ and $1>\Delta(k)>0$. The product of probabilities is $p(\mu, \nu \mid \rho)=p(\mu \mid \rho) p(\nu \mid \rho)$. This is because precise measurements of noncommuting observables must be independently performed on different realizations of the state $\rho$. These events are noncommuting, $[\Delta(\mu), \Delta(v)] \neq 0$. In all the examples to be examined below $\Delta(k)$ are projectors on pure states $\Delta(k)=|k\rangle\langle k|$, so that $p(k \mid \rho)=\langle k|\rho| k\rangle$. In such a case the lack of commutation is expressed as $|\langle\mu \mid v\rangle| \neq 0$.

The product of probabilities $p(\mu, \nu \mid \rho)$ has an upperbound $p\left(\mu, v \mid \Phi_{\mu, v}\right)$ valid for all classical states $\Phi$,

$$
p(\mu, \nu \mid \Phi) \leqslant p\left(\mu, \nu \mid \Phi_{\mu, \nu}\right),
$$

where $\Phi_{\mu, \nu}$ is the classical state that maximizes $p(\mu, \nu \mid \Phi)$. This implies the following nonclassical test:

$$
p(\mu, \nu \mid \rho)>p\left(\mu, \nu \mid \Phi_{\mu, \nu}\right) \longrightarrow \rho \text { is nonclassical. }
$$

Let us comment on this criterion:

(i) As we shall see below, there are actually quantum states providing larger probability than classical states for noncommuting observables.

(ii) The application of these tests crucially depends on the definition of what are classical states. In this work, we adopt the most widely used approach, where a state is classical if its Glauber-Sudarshan phase-space $P$ representation exhibits all the properties of a classical probability density [7]. For nonclassical states, $P$ takes negative values or fails to be a proper function becoming more singular than a delta function. Nevertheless, this is not the unique definition and some other correspondences between states and phase-space 

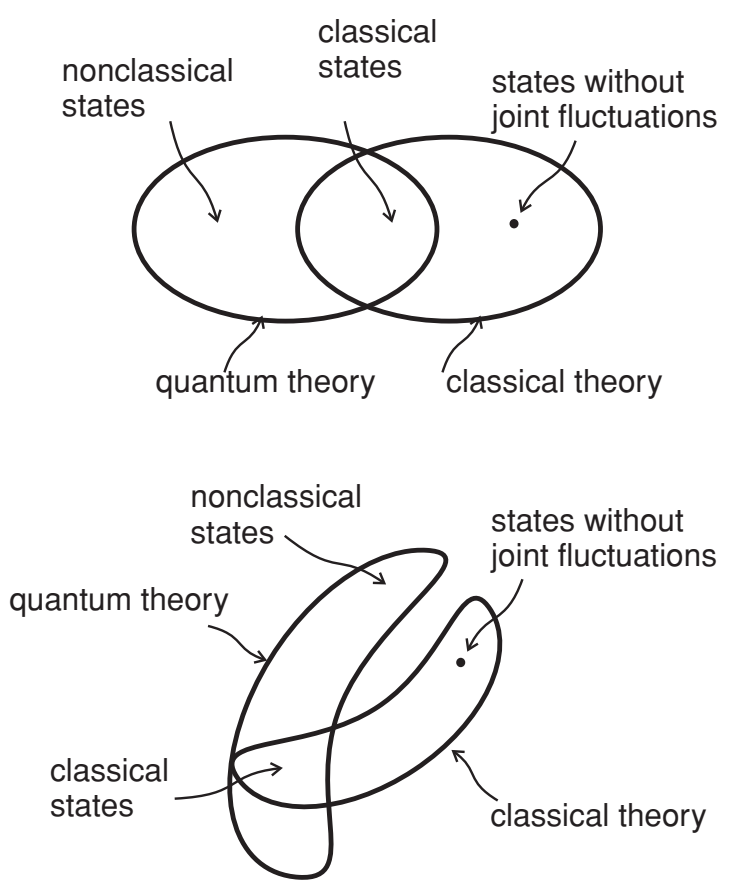

FIG. 1. Diagram sketching in the upper panel the standard intuition regarding the closeness of the classical states within the quantum theory to the states without fluctuations of the classical theory. The results of this work are illustrated in the lower part.

distributions may be adopted [8,9], leading in general to different conclusions [10].

(iii) It holds that

$$
p\left(\mu, \nu \mid \Phi_{\mu, \nu}\right) \leqslant p\left(\mu \mid \Phi_{\mu}\right) p\left(\nu \mid \Phi_{\nu}\right),
$$

where $\Phi_{k}$ are the classical states maximizing $p\left(k \mid \Phi_{k}\right), k=$ $\mu, v$. This is because $p\left(\mu, \nu \mid \Phi_{\mu, \nu}\right)=p\left(\mu \mid \Phi_{\mu, \nu}\right) p\left(\nu \mid \Phi_{\mu, v}\right)$ with $p\left(k \mid \Phi_{\mu, \nu}\right) \leqslant p\left(k \mid \Phi_{k}\right)$ by definition of $\Phi_{k}$. This means that there can be nonclassical product of probabilities that cannot be explained by nonclassicality of the individual statistics. This will hold when $p(\mu, \nu \mid \rho)>p\left(\mu, \nu \mid \Phi_{\mu, \nu}\right)$ with $p(k \mid \rho) \leqslant p\left(k \mid \Phi_{k}\right)$.

(iv) The optimum classical states $\Phi_{\mu, \nu}$ are pure. Classical states $\Phi$ can be expressed as

$$
\Phi=\int d^{2} \alpha P(\alpha)|\alpha\rangle\langle\alpha|, \quad P(\alpha)=p^{2}(\alpha) \geqslant 0,
$$

where $|\alpha\rangle$ are the coherent states, and $p(\alpha)$ is a nonsingular function satisfying the normalization condition $\int d^{2} \alpha p^{2}(\alpha)=$ 1 , and the square is intended to avoid any problem with signs. The product of probabilities can be expressed as

$$
p(\mu, \nu \mid \Phi)=\int d^{2} \alpha d^{2} \beta p^{2}(\alpha) p^{2}(\beta) f_{\mu}(\alpha) f_{v}(\beta),
$$

where $f_{k}(\alpha)=\langle\alpha|\Delta(k)| \alpha\rangle$. Let us maximize $p(\mu, \nu \mid \Phi)$ by varying $p(\alpha) \rightarrow p(\alpha)+\delta p(\alpha)$. This leads to

$$
p(\alpha)\left[f_{\nu}(\alpha) p(\mu \mid \rho)+f_{\mu}(\alpha) p(\nu \mid \rho)\right]=\lambda p(\alpha),
$$

where $\lambda$ is a Lagrange multiplier associated with normalization of the $P$ function. Since $f_{k}(\alpha)$ are not constants, the above equality requires that $p(\alpha)$ must be a delta function centered at some $\alpha_{0}$, being the Lagrange multiplier $\lambda=f_{v}\left(\alpha_{0}\right) p(\mu \mid \rho)+$ $f_{\mu}\left(\alpha_{0}\right) p(\nu \mid \rho)$. Therefore, the optimum classical state must be a pure coherent state $\Phi_{\mu, v}=\left|\alpha_{0}\right\rangle\left\langle\alpha_{0}\right|$.

(v) If $|\mu\rangle,|v\rangle$ are normalized, the maximum of $p(\mu, \nu \mid \rho)$ when $\rho$ runs unrestrictedly all states (including classical and nonclassical) is given by the pure state [11]

$$
|\psi\rangle=\frac{1}{\sqrt{2(1+|\langle\mu \mid \nu\rangle|)}}\left(|\mu\rangle+e^{i \delta}|\nu\rangle\right), \quad \delta=\arg \langle\nu \mid \mu\rangle,
$$

leading to an absolute maximum

$$
p_{\max }(\mu, \nu \mid \rho)=\frac{1}{4}(1+|\langle\mu \mid \nu\rangle|)^{2},
$$

so that for every $\rho$

$$
p(\mu, \nu \mid \rho)=p(\mu \mid \rho) p(\nu \mid \rho) \leqslant \frac{1}{4}(1+|\langle\mu \mid \nu\rangle|)^{2} .
$$

(vi) For pure states $\rho=|\psi\rangle\langle\psi|$, the nonclassicality criterion (3) can be expressed in terms of the modulus of the corresponding Kirkwood phase-space distribution [8],

$$
K(\mu, \nu \mid \rho)=\operatorname{tr}[\rho \Pi(\mu, v)], \quad \Pi(\mu, \nu)=|\mu\rangle\langle\mu \mid v\rangle\langle\nu|,
$$

as

$$
|K(\mu, \nu \mid \rho)|>\left|K\left(\mu, \nu \mid \Phi_{\mu, \nu}\right)\right| \longrightarrow \rho \text { is nonclassical, }
$$
where $\Phi_{\mu, v}$ is the classical pure state that maximizes $|K(\mu, \nu \mid \Phi)|$.

\section{CONJUGATE QUADRATURES}

Let us apply the above approach to a single-mode electromagnetic wave (or, equivalently, to any one-dimensional oscillator) and two values $\mu, \nu=x, y$ of the conjugate field quadratures $X, Y$, (or, equivalently, dimensionless position and momentum) with

$$
X=a^{\dagger}+a, \quad Y=i\left(a^{\dagger}-a\right),
$$

where $a$ is the bosonic annihilation operator, satisfying commutation relations

$$
\left[a, a^{\dagger}\right]=1, \quad[X, Y]=2 i .
$$

The states $|\mu\rangle,|\nu\rangle$ are the unnormalized eigenstates of $X$ and $Y$, respectively, $X|x\rangle=x|x\rangle, Y|y\rangle=y|y\rangle$, with

$$
\langle x \mid y\rangle=\frac{e^{i x y / 2}}{2 \sqrt{\pi}} .
$$

For classical coherent states $|\alpha\rangle$, the quadrature statistics are Gaussian

$$
p(k \mid \alpha)=\frac{1}{\sqrt{2 \pi} \Delta K} \exp \left[-\frac{(k-\langle K\rangle)^{2}}{2(\Delta K)^{2}}\right],
$$

for $k=x, y$ and $K=X, Y$, respectively, being $\langle K\rangle=\langle\alpha|K| \alpha\rangle$ and $(\Delta K)^{2}=1$. The maximum of $p(x, y \mid \alpha)$ is obtained for $\langle X\rangle=x$ and $\langle Y\rangle=y$, such that

$$
p\left(x, y \mid \Phi_{x, y}\right)=\frac{1}{2 \pi}
$$

which holds for $\Phi_{x, y}=\left|\alpha_{0}\right\rangle\left\langle\alpha_{0}\right|$ with $\alpha_{0}=(x+i y) / 2$. In this case, the classical upperbound $p\left(x, y \mid \Phi_{x, y}\right)$ does not depend on $x, y$ and equals the product of upperbounds for the individual events $p\left(x, y \mid \Phi_{x, y}\right)=p\left(x \mid \Phi_{x}\right) p\left(y \mid \Phi_{y}\right)$. 
As an example of nonclassical state violating the above classical bound, let us consider

$$
\rho_{\xi}=\frac{1}{2}\left(\left|\xi_{1}\right\rangle\left\langle\xi_{1}|+| \xi_{2}\right\rangle\left\langle\xi_{2}\right|\right),
$$

where $\left|\xi_{1,2}\right\rangle$ are squeezed states with the same mean values of $X$ and $Y$ :

$$
\left\langle\xi_{\ell}|X| \xi_{\ell}\right\rangle=x, \quad\left\langle\xi_{\ell}|Y| \xi_{\ell}\right\rangle=y, \quad \ell=1,2 .
$$

In this case we have

$$
p\left(x \mid \rho_{\xi}\right)=\frac{1}{2 \sqrt{2 \pi}}\left(\frac{1}{\sigma_{1}}+\frac{1}{\sigma_{2}}\right), \quad p\left(y \mid \rho_{\xi}\right)=\frac{1}{2 \sqrt{2 \pi}}\left(\sigma_{1}+\sigma_{2}\right),
$$

where $\sigma_{\ell}^{2}=\left\langle\xi_{\ell}\left|X^{2}\right| \xi_{\ell}\right\rangle-\left\langle\xi_{\ell}|X| \xi_{\ell}\right\rangle^{2}$. For simplicity, let us consider the case $\sigma_{2}=1 / \sigma_{1}$ so that $p\left(x \mid \rho_{\xi}\right)=p\left(y \mid \rho_{\xi}\right)$ and the product of probabilities is

$$
p\left(x, y \mid \rho_{\xi}\right)=\frac{1}{8 \pi}\left(\frac{1}{\sigma_{1}}+\sigma_{1}\right)^{2} .
$$

This is above the classical upperbound (17) for all $\sigma_{1} \neq 1$. For $\sigma_{\ell}=1$, the states $\left|\xi_{\ell}\right\rangle$ are identical coherent states and therefore classical. Maximum nonclassical behavior holds for $\sigma_{1} \rightarrow 0, \infty$.

Concerning nonclassicality in the individual observables, for $\sigma_{1}=1 / \sigma_{2} \neq 1$ we get

$$
p\left(k \mid \rho_{\xi}\right)>\frac{1}{\sqrt{2 \pi}}=p\left(k \mid \Phi_{k}\right),
$$

where $p\left(k \mid \Phi_{k}\right)$ are the classical upperbounds for the individual quadrature statistics $k=x, y$. This is to say that $\rho_{\xi}$ is nonclassical simultaneously in both conjugate quadratures [2].

Finally, we note that the nonclassical behavior reported here cannot be ascribed in general to the concurrence of other more typical manifestations of nonclassicality [1,5,12]. This is because these states display super-Poissonian number statistics, present no quadrature squeezing, and for large enough $x, y$ they have vanishingly small photon-number oscillations.

\section{PHASE AND NUMBER}

Another distinguished complementary conjugate pair is phase and number. While the number operator is quite straightforward $a^{\dagger} a$, the proper definition of a phase observable may be problematic [13]. Fortunately, there is a large consensus concerning phase states $|\phi\rangle$ that read in the number basis

$$
|\phi\rangle=\frac{1}{\sqrt{2 \pi}} \sum_{n=0}^{\infty} e^{i n \phi}|n\rangle,
$$

defining a suitable phase statistics by $p(\phi \mid \rho)=\langle\phi|\rho| \phi\rangle$. The states $|\phi\rangle$ have infinite norm but provide a legitimate resolution of the identity $I$ since $\int d \phi|\phi\rangle\langle\phi|=I$, so that $p(\phi \mid \rho)$ is always properly normalized. Note that $p(\phi \mid \rho)$ can be larger than one because this is probability density rather than probability, as it usually holds with the distribution of continuous variables, such as position, linear momentum, or field quadratures.

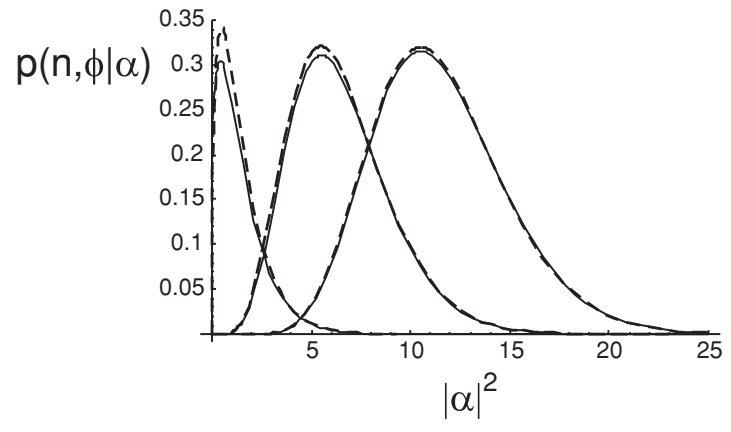

FIG. 2. Plot of the numerical evaluation of the exact $p(n, \phi \mid \alpha)$ for $\arg \alpha=\phi$ (solid line) and its approximation in Eq. (25) (dashed line) for $n=0,5,10$ as functions of $|\alpha|^{2}$.

For classical coherent states $|\alpha\rangle$, we have

$$
p(n \mid \alpha)=\frac{|\alpha|^{2 n}}{n !} e^{-|\alpha|^{2}}, \quad p(\phi \mid \alpha)=\frac{e^{-|\alpha|^{2}}}{2 \pi}\left|\sum_{n=0}^{\infty} \frac{\left(\alpha e^{-i \phi}\right)^{n}}{\sqrt{n} !}\right|^{2} .
$$

The maximum of $p(\phi \mid \alpha)$ holds for $\arg \alpha=\phi$. There is no exact formula for $p(\phi \mid \alpha)$. Nevertheless, there is a simple approximation valid for $|\alpha| \gg 1$ derived after approximating $\alpha^{n} / \sqrt{n}$ ! by a Gaussian function of $n$, assuming $n$ as a continuous variable, leading to $p(\phi \mid \alpha) \simeq \sqrt{2 / \pi}|\alpha|$ when $\arg \alpha=\phi$ [4]. The corresponding product of probabilities is

$$
p(n, \phi \mid \alpha) \simeq \sqrt{\frac{2}{\pi}} \frac{|\alpha|^{2 n+1}}{n !} e^{-|\alpha|^{2}} .
$$

In Fig. 2, we plot the numerical evaluation of the exact $p(n, \phi \mid \alpha)$ for $\arg \alpha=\phi$ (solid line) and its approximation in Eq. (25) (dashed line) for $n=0,5,10$ as functions of $|\alpha|$, showing that the approximation and the exact expression are quite similar even for small $|\alpha|$ and $n$.

The classical distribution (25) has a maximum for $|\alpha|^{2}=$ $n+1 / 2$ leading to the classical upperbound

$$
\begin{aligned}
p\left(n, \phi \mid \Phi_{n, \phi}\right) & \simeq \sqrt{\frac{2}{\pi}} \frac{(n+1 / 2)^{n+1 / 2}}{n !} e^{-(n+1 / 2)} \\
& \simeq \frac{1}{\pi \sqrt{e}}\left(1+\frac{1}{2 n}\right)^{n+1 / 2} \simeq \frac{1}{\pi} e^{1 /(4 n)},
\end{aligned}
$$

which we have simplified by using Stirling approximation. This does not depend on $\phi$ and it depends on $n$ very slightly, decreasing monotonically from its maximum $p\left(n=0, \phi \mid \Phi_{0, \phi}\right)=0.342$ at $n=0$ to the asymptotic limit $\lim _{n \rightarrow \infty} p\left(n, \phi \mid \Phi_{n, \phi}\right)=1 / \pi=0.318$.

The classical upperbound for the product of probabilities does not factorize as the product of individual bounds $p\left(k \mid \Phi_{k}\right)$, $k=n, \phi$,

$$
p\left(n, \phi \mid \Phi_{n, \phi}\right) \neq p\left(n \mid \Phi_{n}\right) p\left(\phi \mid \Phi_{\phi}\right) .
$$

In particular, it holds that $p(\phi \mid \Phi)$ has no maximum for classical states [4].

A first suitable state violating the classical upperbound (26) is the phase coherent state that in the number basis reads [14]

$$
|\varepsilon\rangle=\sqrt{1-|\varepsilon|^{2}} \sum_{n=0}^{\infty} \varepsilon^{n}|n\rangle
$$




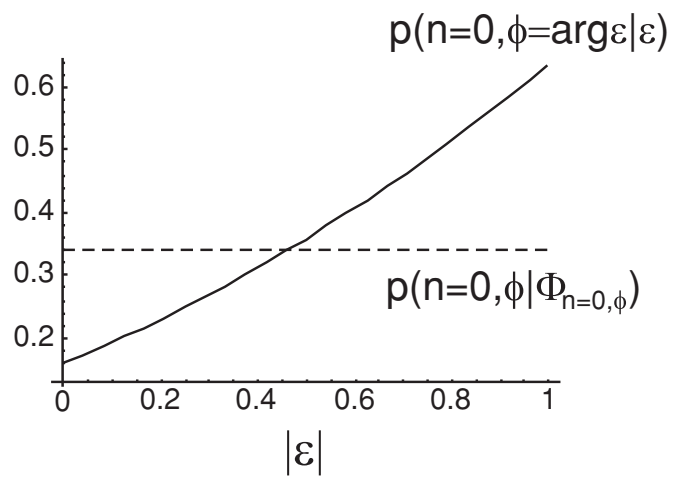

FIG. 3. Plot of $p(n=0, \phi=\arg \varepsilon \mid \varepsilon)$ (solid line) and the classical upperbound (26) $p\left(n=0, \phi \mid \Phi_{n=0, \phi}\right)$ (dashed line) as functions of $|\varepsilon|$ showing that there is nonclassical behavior for $|\varepsilon|>0.47$.

The number statistic is

$$
p(n \mid \varepsilon)=\left(1-|\varepsilon|^{2}\right)|\varepsilon|^{2 n},
$$

while the maximum of the phase distribution $p(\phi \mid \varepsilon)$ occurs for $\phi=\arg \varepsilon$ being

$$
p(\phi=\arg \varepsilon \mid \varepsilon)=\frac{1}{2 \pi} \frac{1+|\varepsilon|}{1-|\varepsilon|} .
$$

The product of probabilities is

$$
p(n, \phi=\arg \varepsilon \mid \varepsilon)=\frac{(1+|\varepsilon|)^{2}}{2 \pi}|\varepsilon|^{2 n} .
$$

In Fig. 3, we have represented $p(n=0, \phi=\arg \varepsilon \mid \varepsilon)$ and the classical upperbound (26) $p\left(n=0, \phi \mid \Phi_{n=0, \phi}\right)$ as functions of $|\varepsilon|$, showing that there is nonclassical behavior for $|\varepsilon|>0.47$. For larger $n$ we get similar plots where the nonclassical behavior holds for larger values of $|\varepsilon|$.

We can provide a more physical picture of how this nonclassical behavior emerges as $|\varepsilon|$ increases by plotting in Fig. 4 separately the number $p(n=0 \mid \varepsilon)$ and phase $p(\phi=\arg \varepsilon \mid \varepsilon)$ probabilities as functions of $|\varepsilon|$. Since they are complementary variables, when one of them increases the other one decreases. The figure shows that the main responsible for breaking the classical bound is the fast increase of the phase probability for increasing $|\varepsilon|$. Moreover, in Fig. 5 we have represented the phase-space picture of $|\varepsilon\rangle$ provided by the $Q$ function,

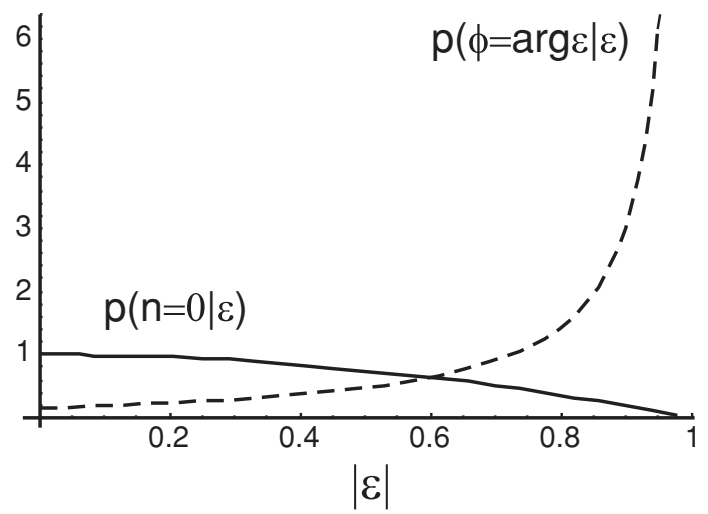

FIG. 4. Plot of the individual number $p(n=0 \mid \varepsilon)$ (solid line) and phase $p(\phi=\arg \varepsilon \mid \varepsilon)$ (dashed line) probabilities for the phase coherent state $|\varepsilon\rangle$ as functions of $|\varepsilon|$.
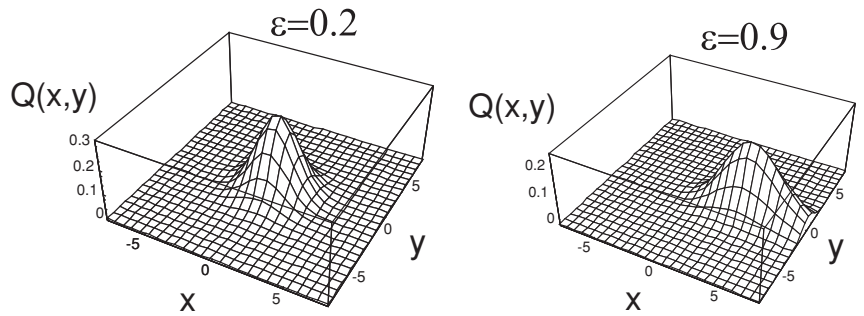

FIG. 5. Plot of the $Q(\alpha)$ function with $\alpha=x / 2+i y / 2$ for a phase coherent state $|\varepsilon\rangle$ with $\varepsilon=0.2$ (left) and $\varepsilon=0.9$ (right) showing that it becomes more elongated as $|\varepsilon|$ increases.

$Q(\alpha)=|\langle\alpha \mid \varepsilon\rangle|^{2} / \pi$ where $|\alpha\rangle$ are coherent states [1], for $\varepsilon=0.2$ and $\varepsilon=0.9$, showing that it becomes more elongated along the $x$ axis as $|\varepsilon|$ increases, reducing phase fluctuations and increasing phase probability.

Phase coherent states are difficult to generate in practice, so let us consider more practical states such as the squeezed coherent states [1],

$$
|\xi\rangle=D(R, \theta) S(r, \varphi)|0\rangle=\sum_{n=0}^{\infty} c_{n}|n\rangle,
$$

where $|0\rangle$ is the vacuum state, being $D$ and $S$, the displacement and squeezing operators, respectively,

$$
D(R, \theta)=e^{R\left(e^{i \theta} a^{\dagger}-e^{-i \theta} a\right)}, \quad S(r, \varphi)=e^{r\left(e^{-i \varphi} a^{2}-e^{i \varphi} a^{\dagger 2}\right) / 2} .
$$

The coefficients of the expansion (32) in the photon-number basis are

$$
\begin{aligned}
c_{n}= & \frac{\left(e^{i \varphi} \tanh r\right)^{\frac{n}{2}}}{\sqrt{2^{n} n ! \cosh r}} \exp \left[-\frac{R^{2}}{2}\left(1+e^{-2 i \delta} \tanh r\right)\right] \\
& \times H_{n}\left[\frac{R}{\sqrt{2}}\left(e^{-i \delta} \sqrt{\tanh r}+\frac{e^{i \delta}}{\sqrt{\tanh r}}\right)\right]
\end{aligned}
$$

where $\delta=\theta-\varphi / 2$ and $H_{n}$ are the Hermite polynomials. The mean number of photons is

$$
\left\langle\xi\left|a^{\dagger} a\right| \xi\right\rangle=R^{2}+\sinh ^{2} r
$$

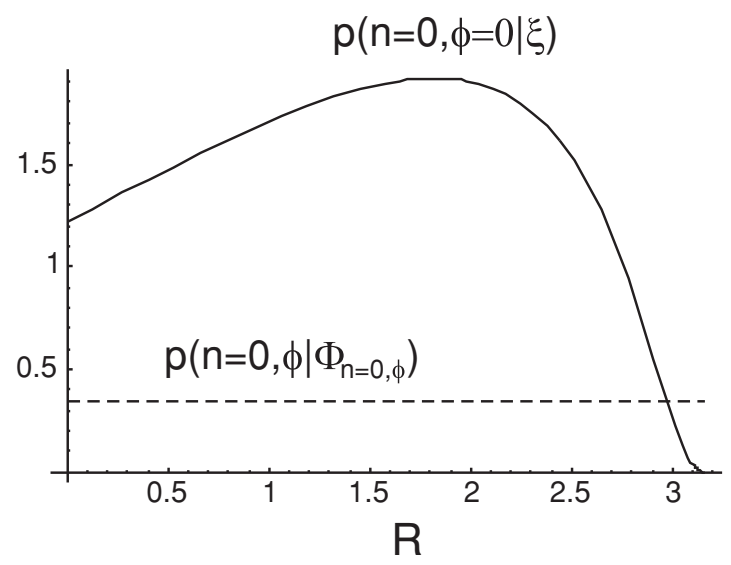

FIG. 6. Plot of $p(n=0, \phi=0 \mid \xi)$ (solid line) and the classical upper bound $p\left(n=0, \phi \mid \Phi_{n=0, \phi}\right)$ (dashed line) as a function of $R$ for a squeezed coherent state with fixed mean number of photons $\left\langle a^{\dagger} a\right\rangle=10$. 


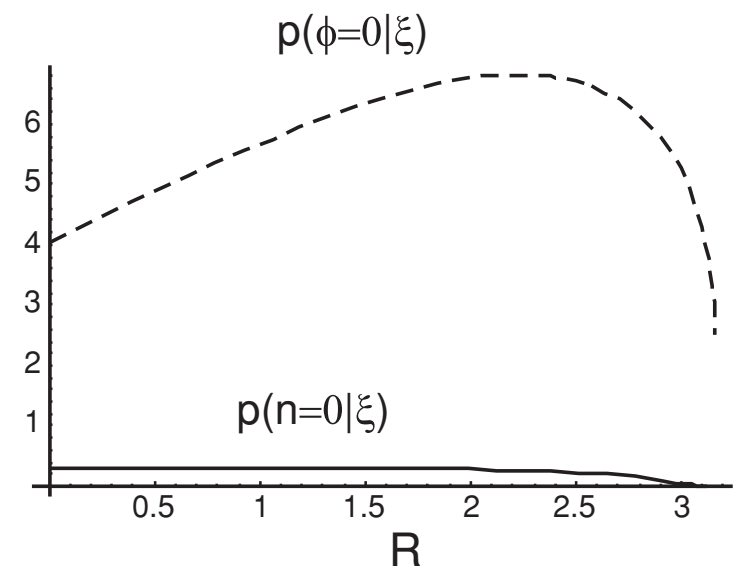

FIG. 7. Plot of the individual number $p(n=0 \mid \xi)$ (solid line) and phase $p(\phi=\arg \xi \mid \xi)$ (dashed line) probabilities for the quadrature squeezed state $|\xi\rangle$ with fixed mean number of photons $\left\langle a^{\dagger} a\right\rangle=10$ as functions of $R$.

In Ref. [15] it has been shown that these states can be very close to phase-coherent states when $\delta= \pm \pi / 2$, which corresponds to the alignment of the major axis of the uncertainty ellipse along the radial direction [16]. Thus, for definiteness we take $\delta=\pi / 2, \theta=0, \varphi=-\pi$.

In Fig. 6 we have represented the product of phase-number probabilities $p(n=0, \phi=0 \mid \xi)$ for a fixed mean number of photons $\left\langle a^{\dagger} a\right\rangle=10$ as a function of $R$, showing that it can be clearly above the classical upperbound $p\left(n=0, \phi \mid \Phi_{n=0, \phi}\right)$ in Eq. (26). The nonclassical behavior disappears when $R \rightarrow$ $\sqrt{\left\langle\xi\left|a^{\dagger} a\right| \xi\right\rangle}$, since in such a case, and for fixed mean photon number, the squeezing disappears $r \rightarrow 0$ and $|\xi\rangle$ tends to be a classical coherent state.

It might be argued that this nonclassical behavior may be a consequence of the oscillatory number distribution of strongly squeezed states [12]. However, this cannot explain the nonclassicality reported in Fig. 6 , since from $R=0$ to $R=2$ the nonclassical behavior increases, while the number oscillation decreases.

Here again we can provide a more physical picture of how this nonclassical behavior is achieved as $R$ increases by plotting in Fig. 7 separately the number $p(n=0 \mid \xi)$ and phase $p(\phi=\arg \xi \mid \xi)$ probabilities as functions of $R$ for a fixed mean number of photons $\left\langle a^{\dagger} a\right\rangle=10$. This shows again that the main responsible for breaking the classical bound is the fast increase of the phase probability overtaking the decrease of the number probability. In Fig. 8 we provide the phase-space
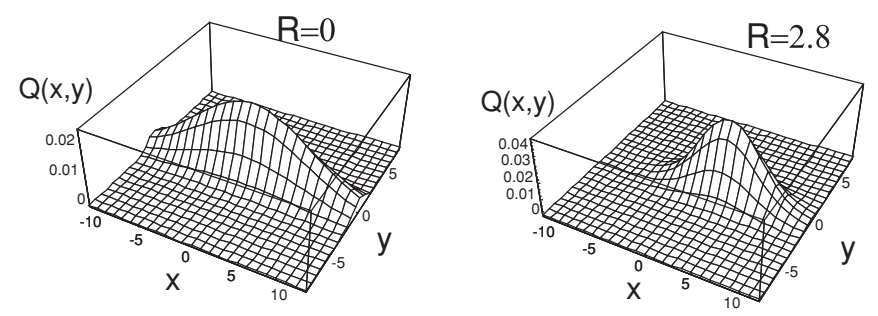

FIG. 8. Plot of the $Q$ function of the squeezed coherent states $|\xi\rangle$ with fixed mean number of photons $\left\langle a^{\dagger} a\right\rangle$ and coherent amplitudes $R=0$ (left) and $R=2.8$ (right). picture of these states for $R=0$ and $R=2.8$, where it can be appreciated that squeezing decreases as the distribution moves away from the origin. Near the origin, the phase distribution has two peaks at $\phi=0, \pi$ [17]. This gives room to increase the phase probability for $\phi=0$ as $R$ increases by favoring the peak at $\phi=0$, despite the fact that simultaneously this reduces squeezing. For $R>2$, the increase of $R$ no longer compensates the decrease of squeezing and both the phase and the product of probabilities decrease. The detailed balance of the phase effects of displacement and squeezing can be clearly appreciated in Fig. 7.

\section{QUADRATURE AND NUMBER}

The main drawback of the above phase-number approach is that there are no simple practical procedures to measure the phase statistics. Because of this we may consider a more practical approach, where the role of the phase may be represented by a suitable quadrature, such as $X$ for example, which can be readily measured by homodyne detection. For coherent states $|\alpha\rangle$, the corresponding quadrature distribution is

$$
p(x)=\frac{1}{\sqrt{2 \pi}} \exp \left[-\frac{(x-\bar{x})^{2}}{2}\right],
$$

where $\bar{x}=\alpha^{*}+\alpha$. For simplicity let us consider compatible events in the sense that $x^{2} \leqslant 4 n$. In such a case the coherent state that maximizes the product of probabilities has $\bar{x}=x$ and $|\alpha|=\sqrt{n}$ leading to

$$
p\left(n, x \mid \Phi_{n, x}\right)=\frac{1}{\sqrt{2 \pi}} \frac{n^{n}}{n !} e^{-n} .
$$

Quadrature squeezed states can beat this upper bound. For example, for $x=n=0$ and squeezed vacuum we have

$$
\begin{aligned}
& p(x=0 \mid \xi)=\frac{1}{\sqrt{2 \pi} \Delta X}=\frac{1}{\sqrt{2 \pi} e^{-r}}, \\
& p(n=0 \mid \xi)=\frac{1}{\cosh r}
\end{aligned}
$$

leading to

$$
p(n=0, x=0 \mid \xi)=\frac{2}{\sqrt{2 \pi}\left(1+e^{-2 r}\right)},
$$

which is above the classical bound $p\left(n=0, x=0 \mid \Phi_{0,0}\right)=$ $1 / \sqrt{2 \pi}$ for all $r>0$.

\section{SPIN OBSERVABLES}

Let us consider a system described by dimensionless angular momentum operators

$$
\left[j_{k}, j_{\ell}\right]=i \sum_{n=1}^{3} \epsilon_{k, \ell, n} j_{n}, \quad\left[j_{0}, \boldsymbol{j}\right]=\mathbf{0}
$$

with

$$
j^{2}=j(j+1)
$$




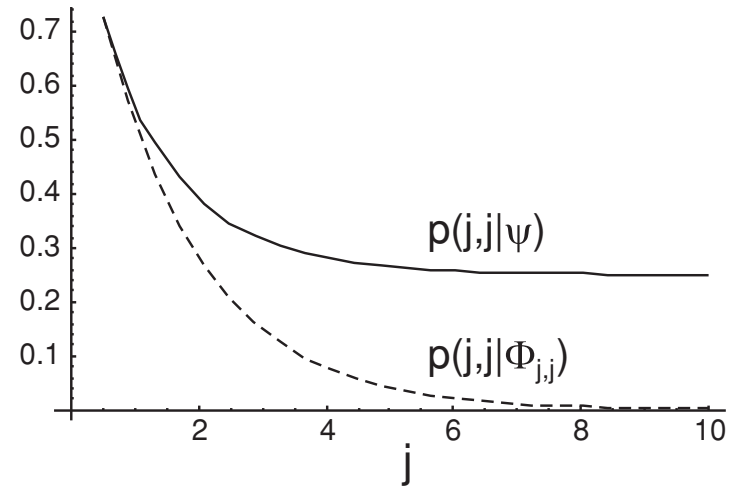

FIG. 9. Plot of the product of probabilities $p(j, j \mid \psi)$ for the state in Eq. (47) (solid line), and the classical upperbound $p\left(j, j \mid \Phi_{j, j}\right)$ in Eq. (46) (dashed line), as functions of $j$ showing nonclassical behavior for $j>1 / 2$.

where $\epsilon_{k, \ell, n}$ is the fully antisymmetric tensor with $\epsilon_{1,2,3}=1$. In the spin context, the classical states are [18]

$$
\Phi=\int d \Omega P(\Omega)|j, \Omega\rangle\langle j, \Omega|, \quad P(\Omega) \geqslant 0,
$$

where $|j, \Omega\rangle$ are the $\mathrm{SU}(2)$ coherent states, which in the basis of eigenvectors of $j^{2}$ and $j_{3}$ read

$$
\begin{aligned}
|j, \Omega\rangle= & \sum_{m=-j}^{j}\left(\begin{array}{c}
2 j \\
j+m
\end{array}\right)^{1 / 2}\left(\sin \frac{\theta}{2}\right)^{j+m} \\
& \times\left(\cos \frac{\theta}{2}\right)^{j-m} e^{i m \varphi}|j, m\rangle_{3},
\end{aligned}
$$

$\Omega=(\theta, \varphi)$ are state parameters, $d \Omega=\sin \theta d \theta d \varphi$, and the subscript in $|j, m\rangle_{k}$ denotes that the vector is eigenstate of $j_{k}$.

For definiteness, let us consider the outcomes $\mu=v=j$ of the noncommuting components $j_{1,2}$ so that $|\mu\rangle=|j, m=j\rangle_{1}$, $|v\rangle=|j, m=j\rangle_{2}$. These observables are always noncommuting, but for $j \neq 1 / 2$ they are not complementary in the sense that $\left.\right|_{1}\left\langle j, m \mid j, m^{\prime}\right\rangle_{2} \mid$ is not constant. The states $|j, j\rangle_{1,2}$ are both equatorial $\mathrm{SU}(2)$ coherent states with $\theta=\pi / 2$ and $\varphi_{1}=0$, $\varphi_{2}=\pi / 2$, respectively, and

$$
\left.\left.\right|_{1}\langle j, j \mid j, j\rangle_{2}\right|^{2}=\frac{1}{2^{2 j}}
$$

The classical state that optimizes the product of probabilities is also an equatorial $\mathrm{SU}(2)$ coherent state $\theta=\pi / 2$, with

$$
p(j, j \mid \theta=\pi / 2, \varphi)=\left(\cos \frac{\varphi}{2} \cos \frac{\pi / 2-\varphi}{2}\right)^{4 j},
$$

which has a maximum for $\varphi=\pi / 4$ leading to the classical upperbound

$$
p\left(j, j \mid \Phi_{j, j}\right)=\left(\cos \frac{\pi}{8}\right)^{8 j} .
$$

Since the $|j, j\rangle_{1,2}$ states are classical the individual upperbounds are trivial $p\left(j \mid \Phi_{j}\right)=1$, so there are no nonclassical states for these outcomes when individually considered since always $p(j \mid \rho) \leqslant p\left(j \mid \Phi_{j}\right)=1$.

In order to proof the existence on nonclassical states violating the classical upperbound (46), let us consider the quantum-optimal state (8)

$$
|\psi\rangle=\frac{1}{\sqrt{2\left(1+\frac{1}{2^{j}}\right)}}\left(|j, j\rangle_{1}+|j, j\rangle_{2}\right),
$$

with

$$
p(j, j \mid \psi)=\frac{1}{4}\left(1+\frac{1}{2^{j}}\right)^{2} .
$$

In Fig. 9, we plot $p(j, j \mid \psi)$ and the classical upperbound $p\left(j, j \mid \Phi_{j, j}\right)$ (46) as functions of $j$ showing nonclassical behavior for $j>1 / 2$ (for $j=1 / 2$ all states are classical).

\section{CONCLUSIONS}

We have derived classical upperbounds for the product of probabilities of two noncommuting events. This discloses nonclassical behavior displayed by the states with probability surpassing the classical bound obeyed by all classical-like states of the quantum theory. We have shown that this holds for some well-known states such as quadrature squeezed and phase-coherent states. To some extent this is paradoxical, since one would expect the classical states of the quantum theory to be closer to the classical theory, where we can have probabilities as close to one as desired simultaneously for any two events.

It might be argued that the results found above follow because increasing the size of the class of states beyond classical states would raise the maximum probability. However, this is not so if the classical class has already reached the ultimate maximum. Precisely this is what actually holds in other closely related situations, since classical coherent states reach both the absolute maximum of Glauber coherence and the absolute minimum of the Heisenberg uncertainty relation for position and momentum or field quadratures. Enlarging the size of the class of states beyond classical states does not improve coherence nor decreases the uncertainty product. Accordingly, one might expect that classical states would reach also the largest product of probabilities allowed by the quantum theory. However, this is not the case as shown above.

\section{ACKNOWLEDGMENTS}

A.L. acknowledges Dr. A. Rivas for enlightening comments. This work has been supported by Project No. FIS200801267 of the Spanish Dirección General de Investigación del Ministerio de Ciencia e Innovación, and by Project QUITEMAD S2009-ESP-1594 of the Consejería de Educación de la Comunidad de Madrid.
[1] L. Mandel and E. Wolf, Optical Coherence and Quantum Optics (Cambridge University Press, Cambridge, England, 1995); M. O. Scully and M. S. Zubairy, Quantum Optics (Cambridge
University Press, Cambridge, England, 1997); H.-A. Bachor, A Guide to Experiments in Quantum Optics (Wiley, Weinheim, Germany, 1998); C. C. Gerry and P. L. Knight, Introductory 
Quantum Optics (Cambridge University Press, Cambridge, England, 2005); V. V. Dodonov, J. Opt. B 4, R1 (2002).

[2] A. Rivas and A. Luis, Phys. Rev. A 79, 042105 (2009).

[3] A. Luis, Phys. Rev. A 82, 024101 (2010).

[4] D. Martín and A. Luis, Phys. Rev. A 82, 033829 (2010).

[5] R. J. Glauber, Phys. Rev. 131, 2766 (1963); H. J. Kimble, M. Dagenais, and L. Mandel, Phys. Rev. Lett. 39, 691 (1977); R. Short and L. Mandel, ibid. 51, 384 (1983); R. E. Slusher, L. W. Hollberg, B. Yurke, J. C. Mertz, and J. F. Valley, ibid. 55, 2409 (1985); L. Mandel, Phys. Scr., T 12, 34 (1986); M. Hillery, Phys. Rev. A 35, 725 (1987); C. T. Lee, ibid. 44, R2775 (1991); 45, 6586 (1992); N. Lütkenhaus and S. M. Barnett, ibid. 51, 3340 (1995); D. N. Klyshko, Phys. Lett. A 213, 7 (1996); W. Vogel, Phys. Rev. Lett. 84, 1849 (2000); Th. Richter and W. Vogel, ibid. 89, 283601 (2002); E. Shchukin, Th. Richter, and W. Vogel, Phys. Rev. A 71, 011802(R) (2005); E. V. Shchukin and W. Vogel, ibid. 72, 043808 (2005); A. Zavatta, V. Parigi, and M. Bellini, ibid. 75, 052106 (2007); R. Alicki and N. Van Ryn, J. Phys. A 41, 062001 (2008); W. Vogel, Phys. Rev. Lett. 100, 013605 (2008); T. Kiesel, W. Vogel, V. Parigi, A. Zavatta, and M. Bellini, Phys. Rev. A 78, 021804(R) (2008); T. Kiesel, W. Vogel, B. Hage, J. DiGuglielmo, A. Samblowski, and R. Schnabel, ibid. 79, 022122 (2009); A. Miranowicz, M. Bartkowiak, X. Wang, Y. X. Liu, and F. Nori, ibid. 82, 013824 (2010); A. Mari, K. Kieling, B. M. Nielsen, E. S. Polzik, and J. Eisert, Phys. Rev. Lett. 106, 010403 (2011).

[6] A. Luis, Phys. Rev. Lett. 88, 230401 (2002).

[7] E. C. G. Sudarshan, Phys. Rev. Lett. 10, 277 (1963); R. J. Glauber, Phys. Rev. 131, 2766 (1963).

[8] J. G. Kirkwood, Phys. Rev. 44, 31 (1933); P. A. M. Dirac, Rev. Mod. Phys. 17, 195 (1945); H.-W. Lee, Phys. Rep. 259, 147 (1995).

[9] H. Margenau and R. N. Hill, Prog. Theor. Phys. 26, 722 (1961); C. L. Mehta, J. Math. Phys. 5, 677 (1964); L. Cohen, ibid. 7, 781 (1966); K. E. Cahill and R. J. Glauber, Phys. Rev. 177, 1857 (1969); 177, 1882 (1969); G. S. Agarwal and E. Wolf, Phys. Rev. D 2, 2161 (1970); 2, 2187 (1970); 2, 2206 (1970).
[10] J. A. Vaccaro, Opt. Commun. 113, 421 (1995); Phys. Rev. A 52, 3474 (1995); J. A. Vaccaro and D. T. Pegg, ibid. 41, 5156 (1990); L. M. Johansen, Phys. Lett. A 329, 184 (2004); J. Opt. B 6, L21 (2004); L. M. Johansen and A. Luis, Phys. Rev. A 70, 052115 (2004); A. Luis, ibid. 73, 063806 (2006).

[11] A. Luis, Phys. Rev. A 80, 034101 (2009).

[12] W. Schleich and J. A. Wheeler, Nature (London) 326, 574 (1987); J. Opt. Soc. Am. B 4, 1715 (1987); J. Peřina and J. Bajer, Phys. Rev. A 41, 516 (1990).

[13] P. Carruthers and M. M. Nieto, Rev. Mod. Phys. 40, 411 (1968); D. T. Pegg and S. M. Barnett, J. Mod. Opt. 44, 225 (1997), Special issue on quantum phase and phase dependent measurements, Phys. Scr. T 48, (1993), edited by W. Schleich and S. M. Barnett; A. Luis and L. L. Sánchez-Soto, in: E. Wolf (Ed.) Progress in Optics, Vol. 41 (Elsevier, Amsterdam, 2000), p. 421; Phys. Rev. A 48, 4702 (1993); R. Lynch, Phys. Rep. 256, 367 (1995); A. Luis, L. L. Sánchez-Soto, and R. Tanaś, Phys. Rev. A 51, 1634 (1995); A. Luis and L. L. Sánchez-Soto, ibid. 53, 495 (1996); R. Tanaś, A. Miranowicz, and T. Gantsog, in: E. Wolf (Ed.) Progress in Optics, Vol. 35 (Elsevier, Amsterdam, 1996), p. 355; V. Peřinová, A. Lukš, and J. Peřina, Phase in Optics (World Scientific, Singapore, 1998).

[14] E. C. Lerner, H. W. Huang, and G. E. Walters, J. Math. Phys. 11, 1679 (1970); A. Vourdas, Phys. Rev. A 45, 1943 (1992); E. C. G. Sudarshan, Int. J. Theor. Phys. 32, 1069 (1993); A. Vourdas, Phys. Scr., T 48, 84 (1993); A. Vourdas, C. Brif, and A. Mann, J. Phys. A 29, 5887 (1996); A. Vourdas, J. Phys. A 39, R65 (2006); C. C. Gerry and T. Bui, Phys. Rev. A 80, 033831 (2009).

[15] A. Luis, Phys. Lett. A 354, 71 (2006).

[16] M. Freyberger and W. Schleich, Phys. Rev. A 49, 5056 (1994); A. Aragão, A. T. Avelar, and B. Baseia, Phys. Lett. A 331, 366 (2004); A. Aragão, P. B. Monteiro, A. T. Avelar, and B. Baseia, ibid. 337, 296 (2005).

[17] W. Schleich, R. J. Horowicz, and S. Varro, Phys. Rev. A 40, 7405 (1989).

[18] F. T. Arecchi, E. Courtens, R. Gilmore, and H. Thomas, Phys. Rev. A 6, 2211 (1972); O. Giraud, P. Braun, and D. Braun, ibid. 78, 042112 (2008). 